\title{
PILARIZAÇÃo DE UMA ARGILA BRASILEIRA COM POLIIDROXICATIONS DE ALUMÍNIO: PREPARAÇÃO, CARACTERIZAÇÃo E PROPRIEDADES CATALÍTICAS
}

\author{
Sibele Berenice Castellã Pergher* \\ Departamento de Química, Universidade Regional Integrada do Alto Uruguai e das Missões - Campus Erechim, CP 743, \\ 99700-000 Erechim - RS \\ Renato Sprung \\ Departamento de Processos Químicos, Faculdade de Engenharia Química, Universidade Estadual de Campinas, Campinas - SP
}

Recebido em 4/5/04; aceito em 10/12/04; publicado na web em 25/5/05

\begin{abstract}
PILLARIZATION OF A BRAZILIAN CLAY WITH ALUMINIUM POLYHYDROXICATIONS: PREPARATION, CHARACTERIZATION AND CATALYTIC PROPERTIES. Montmorillonite clay from Brazil was pillared with aluminium polyhydroxications. The influence of aging of the pillaring solution and the concentration of the clay suspension on the properties of the prepared materials was studied. The materials were characterized by chemical analysis, XRD and pore analysis by $\mathrm{N}_{2}$ adsorption. The catalytic properties were evaluated in the cumene cracking reaction. Results showed that the pillarization process increases the basal spaces of natural clay from 9.7 to $18.5 \AA$ and the surface area from 41 to $300 \mathrm{~m}^{2} / \mathrm{g}$.
\end{abstract}

Keywords: pillared clays; montmorillonite; aluminium polyhydroxication.

\section{INTRODUÇÃO}

As argilas pilarizadas são um dos grupos de materiais mais estudados dentro dos grupos de materiais micro e mesoporosos desenvolvidos pela engenharia molecular. $\mathrm{O}$ interesse por argilas pilarizadas surge no desenvolvimento de "Fluid Cracking catalysts" (FCC) que sejam capazes de converter moléculas grandes existentes nas frações pesadas dos óleos. As zeólitas, o FCC tradicional, contém poros relativamente pequenos de 6 a $9 \AA$, por isso requerem um préprocessamento das moléculas grandes antes de serem utilizadas. Vários estudos vêm sendo realizados na preparação de zeólitas com poros maiores ou com grande acessibilidade. Uma alternativa é a utlização de argilas pilarizadas que podem ter poros maiores que as zeólitas.

As argilas pilarizadas são sintetizadas mediante a troca iônica de cátions da argila, como $\mathrm{Ca}^{+2}$ e $\mathrm{Na}^{+}$, por cátions maiores, como por ex. poliidroxicátions de $\mathrm{Al}, \mathrm{Zr}$, Ti e outros. $\mathrm{O}$ material resultante, depois da calcinação, contém óxidos que funcionam como pilares, mantendo as camadas separadas e expondo as superfícies internas das argilas. As dimensões das cavidades podem ser significativamente maiores que as produzidas nas zeólitas e a acidez é suficiente para catalisar reações de craqueamento. Pela variação do tamanho do pilar e/ou do espaçamento entre pilares, pode-se ter um tamanho de poro adequado para uma determinada aplicação, que não se restringe ao craqueamento catalítico.

A bibliografia de preparação e aplicação das argilas pilarizadas é bastante ampla e algumas revisões têm sido apresentadas ${ }^{1-8}$.

O presente trabalho relata a viabilidade da preparação e o estudo de algumas propriedades de uma argila Montmorillonita paranaense pilarizada com oligômeros de alumínio.

\section{PARTE EXPERIMENTAL}

A argila utilizada foi uma Montmorillonita da região de São Mateus do Sul (PR - Brasil), facilitada pela PETROSIX, sem tratamentos adicionais ${ }^{4}$. O material foi pilarizado com oligômeros de

*e-mail: pergher@uri.com.br alumínio e estudou-se a influência da concentração da suspensão da argila e o tempo de oligomerização nas suas propriedades. Os materiais preparados foram avaliados cataliticamente frente ao craqueamento do Cumeno.

A preparação das argilas pilarizadas consiste basicamente em três etapas: preparação da solução pilarizante; tratamento da argila com a solução pilarizante e calcinação do material sólido obtido

$\mathrm{O}$ agente pilarizante utilizado foi uma solução de oligômeros de alumínio preparada pela adição lenta de $\mathrm{NaOH}(0,2 \mathrm{~mol} / \mathrm{L})$ a uma solução de $\mathrm{AlCl}_{3} \cdot 6 \mathrm{H}_{2} \mathrm{O}(0,2 \mathrm{~mol} / \mathrm{L})$ sob agitação contínua, até alcançar uma relação molar de $\mathrm{OH} / \mathrm{Al}=2,0$ e envelhecida durante $1 \mathrm{~h}, 6$ ou 15 dias à temperatura ambiente.

A argila foi tratada com a solução pilarizante preparada em quantidade equivalente à relação Al/Mont de $15 \mathrm{meq} \mathrm{Al} / \mathrm{g}$ de argila, durante $2 \mathrm{~h}$ a $25{ }^{\circ} \mathrm{C}$ e em agitação. Nesta etapa a argila foi utilizada nas seguintes formas: seca, suspensão de $1 \mathrm{~g} / 15 \mathrm{~mL}$ de $\mathrm{H}_{2} \mathrm{O}$ e suspensão de $1 \mathrm{~g} / 100 \mathrm{~mL}$ de $\mathrm{H}_{2} \mathrm{O}$.

$\mathrm{O}$ material resultante, depois de filtrado, lavado e seco à temperatura ambiente, foi calcinado da seguinte forma: $0,5 \mathrm{~h}$ a $150{ }^{\circ} \mathrm{C}$ e $2,5 \mathrm{~h}$ a $450{ }^{\circ} \mathrm{C}$.

Os métodos utilizados para a caracterização dos materiais sólidos preparados foram análises química, difração de raios $\mathrm{X}$ e propriedades texturais por adsorção de nitrogênio.

\section{Análise química}

A análise da composição das argilas foi realizada por fluorescência de raios X (FRX). Para esta análise, a argila é misturada com um fundente (mistura de tetraborato e metaborato de lítio), numa relação em massa de 1:5 (argila:fundente). Esta mistura é homogeneizada e, a seguir, são adicionadas $0,3 \mathrm{~mL}$ de solução de $\mathrm{LiBr}$ a $4 \%$ em peso. $\mathrm{O}$ cadinho contendo a mistura é levado à fusão num forno Rigaku a $1200{ }^{\circ} \mathrm{C}$, por aproximadamente $7 \mathrm{~min}$. A pastilha formada é analisada utilizando-se um espectro de raios $\mathrm{X}$ Philips modelo PW 1480. Este equipamento possui um tubo gerador de raios $\mathrm{X}$ de ródio. As medidas foram realizadas no CENPES/ PETROBRÁS. 


\section{Difração de raios $X$}

O equipamento utilizado foi um difratômetro Philips X'Pert PW3719 equipado com um polarizador de grafite, janelas automáticas com superfície total de varredura constante de $14 \mathrm{~mm}$, portaamostras giratório, câmara de temperatura Anton Parr modelo HTK16 e detector proporcional. Utilizou-se a radiação K $\alpha$ do Cobre $(\lambda=1,5418 \AA$ ), e uma potência de excitação de $2 \mathrm{~kW}$. A velocidade angular do goniômetro foi de $0,02 \%$, e o intervalo usual de varredura foi de $2 \theta=1$ a $40^{\circ}$.

\section{Propriedades texturais por adsorção de nitrogênio}

Para a obtenção dos dados de adsorção foram utilizados dois equipamentos: o medidor de área superficial CG 2000 e o Micromeritcs ASAP 2400. Medidor de área superficial CG2000: pesou-se em torno de $10 \mathrm{mg}$ da amostra e com uma corrente de $\mathrm{N}_{2}$ era pré-tratada por $2 \mathrm{~h}$ à $250{ }^{\circ} \mathrm{C}$. Depois do pré-tratamento, realizavam-se as medições de adsorção de $\mathrm{N}_{2}$ na temperatura do nitrogênio líquido. Para as adsorções utilizou-se duas misturas de gases de $\mathrm{He} / \mathrm{N}_{2}$, uma com $5 \%$ de $\mathrm{N}_{2}$, que foi utilizado para medidas de adsorção para $\mathrm{p} / \mathrm{p}_{\mathrm{o}}$ até 0,35 , e outra com $15 \%$ de $\mathrm{N}_{2}$, que foi utilizada para as medidas de adsorção para $\mathrm{p} / \mathrm{p}$ o maiores que 0,30 . As medidas foram realizadas no Depto. de Engenharia Química, da Universidade Estadual de Maringá. Micromeritcs ASAP 2400: amostras com aproximadamente $200 \mathrm{mg}$ foram pré-tratadas a $200{ }^{\circ} \mathrm{C}$ à vácuo durante $4 \mathrm{~h}$, sendo feita, então, a adsorção de $\mathrm{N}_{2}$ à temperatura do nitrogênio líquido. As medidas foram realizadas no CENPES/PETROBRÁS.

\section{Avaliação catalítica}

Os materiais preparados e caracterizados foram avaliados através da reação de craqueamento do cumeno. Os testes catalíticos foram conduzidos num minireator de aço inox contendo $0,4 \mathrm{~g}$ de argila diluída em $1,2 \mathrm{~g}$ de areia. $\mathrm{O}$ cumeno foi alimentado à taxa de $3,15 \mathrm{~g} / \mathrm{h}$, utilizando-se uma vazão de $20 \mathrm{~mL}$ de $\mathrm{N}_{2} /$ min como gás de arraste. Os produtos líquidos foram condensados a $6-7{ }^{\circ} \mathrm{C}$ na saída do reator, pesados e analisados em cromatógrafo de gás Varian com coluna Apierozon-L, a $135^{\circ} \mathrm{C}$ e vazão de $20 \mathrm{~mL} / \mathrm{min}$ de $\mathrm{N}_{2}$. Os resultados são representados em $\mathrm{g}$ de benzeno produzidos em 60 min de reação.

\section{RESULTADOS E DISCUSSÃO}

A argila natural foi denominada Mnat e os materiais preparados foram denominados MPx:yd, sendo x o valor da concentração da suspensão de argila e y o tempo de envelhecimento da solução pilarizante utilizados na preparação.

Através da análise química (Tabela 1) observa-se que a argila possui $\mathrm{Ca}^{+2}$ como cátion interlamelar e que também contém Fe, $\mathrm{K}$, $\mathrm{Mg}$ e Mn. Ao pilarizar-se a argila natural, ocorre uma perda de
96-98\% do teor de Ca e aumentos consideráveis no teor de Al, indicando que ocorreu a troca cationica de Ca pelos oligômeros de alumínio.

Observa-se também uma redução nos teores de $\mathrm{Fe}_{2} \mathrm{O}_{3}, \mathrm{MgO}$ e $\mathrm{Mn}_{2} \mathrm{O}_{3}$, o que aparentemente indica que parte dos íons $\mathrm{Fe}, \mathrm{Mg}$ e Mn também participam do processo e deveriam estar localizados no espaço interlaminar. A substituição dos ions $\mathrm{K}$ é pequena.

A Figura 1 mostra a variação da quantidade de alumínio incorporada em função da concentração da suspensão e do tempo de envelhecimento da solução pilarizante. Observa-se que um aumento da diluição da suspensão de argila proporciona um acréscimo na quantidade de alumínio incorporado. O processo de suspensão facilita a intercalação dos oligômeros. Observa-se também que o aumento do tempo de oligomerização resulta em uma diminuição da quantidade de alumínio incorporado, o que pode estar associado ao tamanho dos oligômeros. A argila incorpora cerca de $15 \%$ mais de alumínio de soluções envelhecidas por $1 \mathrm{~h}$ que soluções envelhecidas por 15 dias.

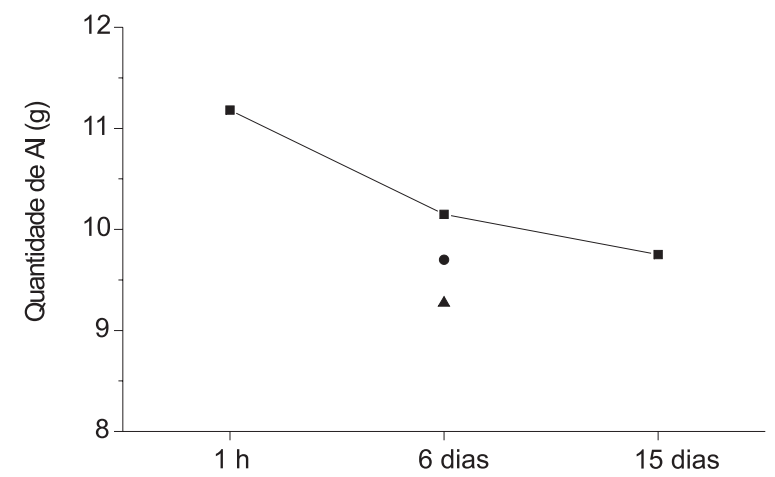

Figura 1. Quantidade de alumínio incorporado em função da concentração da suspensão e do tempo de envelhecimento da solução pilarizante $(\boldsymbol{\Lambda}=$ $1 \mathrm{~g} / 0 \mathrm{~mL} ; \bullet=1 \mathrm{~g} / 15 \mathrm{~mL} ; \mathbf{\square}=1 \mathrm{~g} / 100 \mathrm{~mL}$ )

Os difractogramas de raios-X (em pó) da argila natural e de uma argila pilarizada, ambas sem calcinar estão representados na Figura 2. Confirma-se pelo difratograma que é uma argila montmorillonita e que contém, provavelmente, quartzo como impureza. Observa-se um deslocamento da primeira banda (001) a valores menores de $2 \theta$, indicando que o processo de incorporação de $\mathrm{Al}$ na argila natural originou um material com um espaçamento basal de $\sim 18,5 \AA$, que se mantém depois de calcinar a $450{ }^{\circ} \mathrm{C}$. Este aumento do espaçamento basal de $\sim 3 \AA$ ou $\sim 8,5 \AA$ (se compararmos com a argila desidratada) é um indicativo de que as lâminas do material se encontram separadas por pilares de Al. Pode-se ver que as outras bandas correspondentes à estrutura da montmorillonita se mantêm, confirmando que as lâminas não sofrem modificações durante o processo.

Observa-se que na argila pilarizada aparecem duas bandas a baixos ângulos, uma correspondente ao espaçamento basal de $\sim 18,5 \AA$

Tabela 1. Resultados da análise química em g, tomando-se a argila natural como base de cálculo (100 g)

\begin{tabular}{lcccccccccccc}
\hline & $\mathrm{Al}_{2} \mathrm{O}_{3}$ & $\mathrm{SiO}_{2}$ & $\mathrm{CaO}$ & $\mathrm{Fe}_{2} \mathrm{O}_{3}$ & $\mathrm{~K}_{2} \mathrm{O}$ & $\mathrm{SO}_{3}$ & $\mathrm{MgO}$ & $\mathrm{Mn}_{2} \mathrm{O}_{3}$ & $\mathrm{TiO}_{2}$ & $\mathrm{P}_{2} \mathrm{O}_{5}$ & $\mathrm{Total}$ \\
\hline MNat. & 23,01 & 60,86 & 2,29 & 5,60 & 1,10 & 0,2 & 6,49 & 0,11 & 0,29 & 0,06 & 100 \\
MP0:6d & 32,28 & 60,86 & 0,063 & 4,98 & 1,04 & 0,42 & 5,83 & 0,053 & 0,28 & 0,063 & 105,8 \\
MP15:6d & 32,71 & 60,86 & 0,053 & 5,06 & 1,01 & 0,64 & 5,98 & 0,053 & 0,28 & 0,064 & 106,7 \\
MP100:0d & 34,19 & 60,86 & 0,065 & 5,27 & 0,99 & 0,32 & 5,93 & 0,065 & 0,28 & 0,054 & 108,0 \\
MP100:6d & 33,16 & 60,86 & 0,053 & 4,84 & 0,99 & $<0,1$ & 6,07 & 0,053 & 0,28 & 0,064 & 106,4 \\
MP100:15d & 32,76 & 60,86 & 0,053 & 5,06 & 1,02 & 0,21 & 5,85 & 0,053 & 0,28 & 0,053 & 106,2 \\
\hline
\end{tabular}



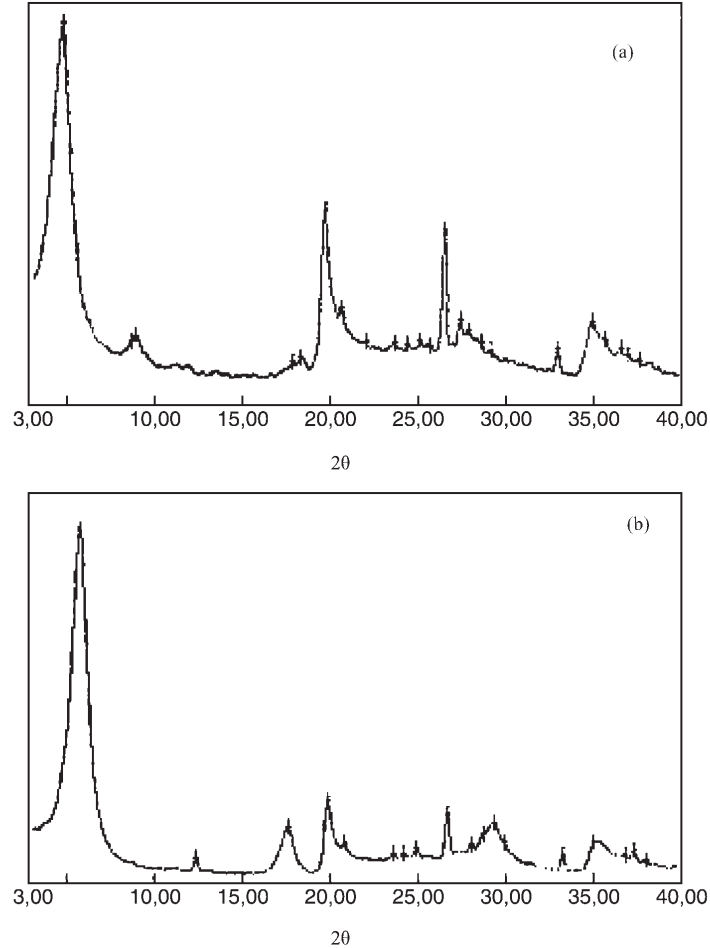

Figura 2. Difractograma de raios- $X$ da argila pilarizada (MP100:6d) (a) $e$ argila natural (b), ambas sem calcinar

e outra menos intensa a $\sim 9,7 \AA$. Este espaçamento de $\sim 9,7 \AA$ é característico da argila calcinada, onde as lâminas se encontram colapsadas. Poderia ocorrer que o tratamento de troca catiônica dos oligômeros de $\mathrm{Al}$ com os cátions interlaminares $\left(\mathrm{Ca}^{+2}\right)$ formasse um material composto de duas partes, uma pilarizada com espaçamento de $18,5 \AA$ e outra colapsada com espaçamento de $9,7 \AA$ (ver ref. 9 onde se discute a presença desta banda e a distribuições dos pilares).

Através dos difractogramas de raios-X pode-se observar, também, o grau de distribuição dos pilares mediante a intensidade e largura da banda correspondente ao plano (001). Em estudos anteriores $^{9}$ observa-se que com o aumento da relação Al/Mont ocorre um aumento da intensidade da banda 001 até a relação $\mathrm{Al} / \mathrm{mont}=$ 15 , acima da qual se mantém. Isto significa que para obter uma boa distribuição de pilares, a relação Al/Mont mínima requerida é 15. Também observa-se que a temperatura de calcinação influi no grau de pilarização do material. Com o aumento da temperatura de calcinação, há uma diminuição de intensidade da banda 001 , a $750{ }^{\circ} \mathrm{C}$ a estrutura já apresenta sinais de ruptura e a $900{ }^{\circ} \mathrm{C}$ está totalmente destruída. Estes resultados sugeriram que uma temperatura de calcinação correta seria $450{ }^{\circ} \mathrm{C}$.

Os efeitos das condições de preparação sobre os espaçamentos basais dos materiais pilarizados são apresentados na Figura 3. Os valores referem-se a produtos calcinados a $450{ }^{\circ} \mathrm{C}$. Estes espaçamentos podem ser comparados às quantidades de oligômeros incorporados na argila apresentados na Figura 1.

Para um tempo de oligomerização de 6 dias, o aumento do grau de diluição da suspensão de argila resultou em acréscimos tanto do espaçamento basal como na quantidade de oligômeros incorporados ao material pilarizado. Aumentando-se o tempo de oligomerização para 15 dias, reduziu-se tanto o espaçamento basal como a incorporação de oligômeros. Nos dois casos existe uma relação direta entre os aumentos da quantidade de oligômeros incorporados e do espaçamento basal. Esta relação desapareceu quando o tempo de oligomerização foi diminuído para $1 \mathrm{~h}$. O material



Figura 3. Espaçamento Basal em função da concentração da suspensão de argila e tempo de envelhecimento da solução pilarizante $(\mathbf{\Delta}=1 \mathrm{~g} / 0 \mathrm{~mL}$; $=1 \mathrm{~g} / 15 \mathrm{~mL} ; \mathbf{\square}=1 \mathrm{~g} / 100 \mathrm{~mL}$ )

MP100:0d apresentou uma diminuição significativa do espaçamento basal em relação à MP100:6d, de 18,2 Å para 17,3 A. No entanto, a quantidade de oligômeros incorporados aumentou. Neste caso parece claro que houve uma incorporação significativa de oligômeros menores.

A Figura 4 mostra as isotermas de adsorção de $\mathrm{N}_{2}$ da argila natural e da amostra pilarizada PM100:6d (calcinada a $500{ }^{\circ} \mathrm{C}$ ) empregando o CG2000. Observa-se que, com o tratamento da argila natural com a solução oligomérica, ocorre um aumento considerável da quantidade de $\mathrm{N}_{2}$ adsorvido. Para p/po $\sim 0,2$ estes valores são 10,2 na argila natural e 103,3 $\mathrm{cm}^{3} / \mathrm{g}$ na amostra PM100:6d, um aumento de 10 vezes na quantidade de $\mathrm{N}_{2}$ adsorvido.

Sabe-se de análises anteriores que ao tratar a argila natural com a solução oligomérica, ocorre uma incorporação de Al e um aumento no espaçamento basal de 9,8 a $\sim 18 \AA$. Estes resultados evidenciam que os oligômeros atuam como pilares, separando as lâminas em cerca de $8 \AA$, e propiciam um maior acesso de moléculas menores às superfícies internas dos materiais. Como é o caso do $\mathrm{N}_{2}$, cujo tamanho pode ser avaliado pelo diâmetro cinético de 3,64 $\AA$ ou pelo diâmetro molecular máximo de $4,09 \AA^{10}$. Este espaçamento interlaminar de 8 Å é característico das dimensões de microporos, o que justifica a grande adsorção de $\mathrm{N}_{2}$ para baixos p/po e a isoterma do tipo I observados na Figura 4.

As quantidades de $\mathrm{N}_{2}$ adsorvidas foram convertidas em área específica, utilizando-se o método BET. Os resultados estão na Tabela 2 e na Figura 5.

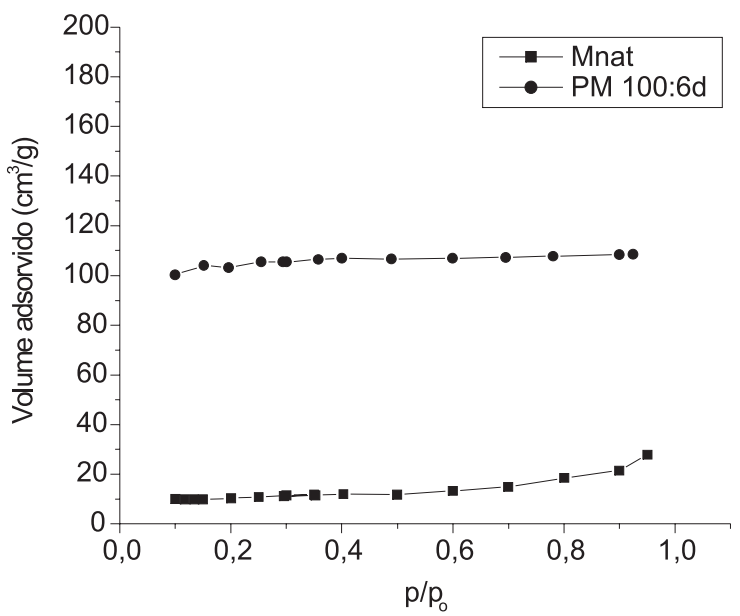

Figura 4. Isotermas de adsorção de $N_{2}$ da Mnat e PM 100:6d pelo método CG2000 


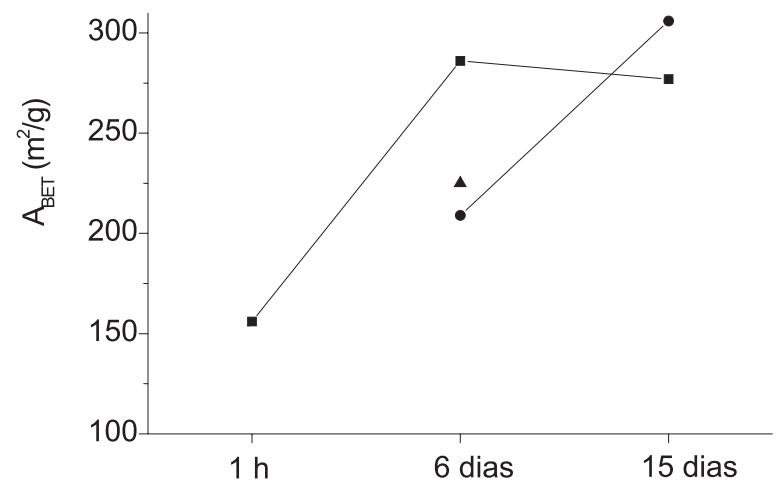

Figura 5. Superfícies específicas BET em função da concentração da suspensão de argila e tempo de envelhecimento da solução pilarizante $(\mathbf{\Lambda}=$ $1 \mathrm{~g} / 0 \mathrm{~mL} ; \mathbf{O}=1 \mathrm{~g} / 15 \mathrm{~mL} ; \mathbf{\square}=1 \mathrm{~g} / 100 \mathrm{~mL}$ )

Para a argila natural obtiveram-se áreas de 33,3 e 45,8 $\mathrm{m}^{2} / \mathrm{g}$ antes e após calcinação a $450{ }^{\circ} \mathrm{C}$. A área da amostra pilarizada PM100:6d calcinada a $500{ }^{\circ} \mathrm{C}$ foi cerca de 10 vezes superior à da argila natural, $324 \mathrm{~m}^{2} / \mathrm{g}$, relação semelhante à quantidade de $\mathrm{N}_{2}$ adsorvido.

Observando os resultados da Tabela 2, nota-se que a amostra pilarizada PM100:0d possui a menor área $\sim 156 \mathrm{~m}^{2} / \mathrm{g}$; estes valores correspondem aproximadamente à metade dos valores obtidos para outras amostras pilarizadas. A difração de raios $\mathrm{X}$ mostrou que a PM100:0d possui um $\mathrm{d}_{001}$ de $17,3 \AA$, que é apenas 0,5 a $0,9 \AA$ menor que o das outras amostras, e não justifica diretamente um aumento tão significativo de área. A quantidade de $\mathrm{Al}$ incorporado na PM100:0d é 15\% maior que nos outros materiais pilarizados, o que também não justifica as diferenças nas áreas.

A distância interlaminar de PM100:0d, diferença entre o seu $\mathrm{d}_{001}$ e o da argila calcinada, é de $7,4 \AA$, enquanto que para outros materiais pilarizados varia de $\sim 7,9$ a 8,3 A. Estas distâncias interlaminares são suficientes para o acesso de moléculas de $\mathrm{N}_{2}$ de diâmetro entre 3,6 a 4,1 $\AA$ e, certamente, ocorre a adsorção de uma monocamada de $\mathrm{N}_{2}$ em todas as amostras. O desconhecimento das dimensões reais da molécula de $\mathrm{N}_{2}$ e do seu empacotamento levam a incertezas sobre a possibilidade de adsorção de duas camadas de $\mathrm{N}_{2}$ no espaço interlaminar. A espessura de duas camadas de $\mathrm{N}_{2}$ pode variar entre 7,3 a $8,2 \AA$, o que está na mesma ordem de grandeza da distância interlaminar. As áreas superiores a $155 \mathrm{~m}^{2} / \mathrm{g}$ podem, portanto, representar diferentes graus de adsorção de $\mathrm{N}_{2}$ em dupla camada nos materiais.

$\mathrm{O}$ grau de adsorção de $\mathrm{N}_{2}$ em dupla camada seria, nestas condições, muito sensível a pequenas variações de espaçamento basal.

As isotermas de adsorção de $\mathrm{N}_{2}$, obtidas pelo ASAP 2400, da argila natural e de uma argila pilarizada são representadas na Figura 6. A isoterma de adsorção de $\mathrm{N}_{2}$ corresponde a uma isoterma do tipo IV ${ }^{11}$, característica de materiais mesoporosos, podendo ou não conter microporos. $\mathrm{O}$ ciclo de histerese é do tipo $\mathrm{B}^{11}$, típico de poros tipo fenda ("Slit-Shape Pores"). Neste tipo de poros, a adsorção ocorre pela formação de multicamadas, enquanto que a dessorção, por evaporação capilar.

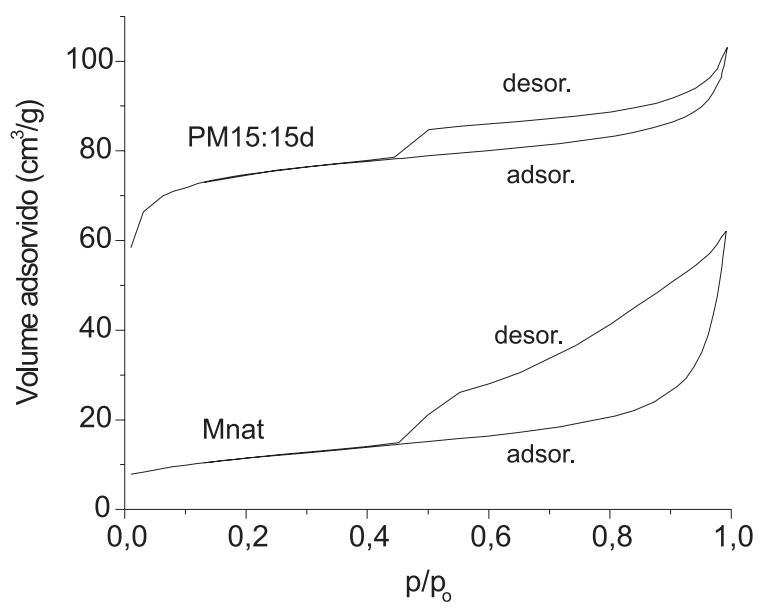

Figura 6. Isotermas de adsorção de $N_{2}$ das amostras: argila natural e PM15:15d

A partir dos dados da isoterma calculam-se a superficie específica e o volume de poro ${ }^{11}$. A superficie específica total calcula-se segundo o método BET e o volume total é calculado a uma pressão parcial $\mathrm{p} / \mathrm{po}=0,98$. A superfície específica e o volume de microporo calculam-se pelo método t-plot. A superfície externa é a diferença entre a BET e a do microporo, que compreende a contribuição de meso e macroporos. O volume de mesoporos calcula-se pelo método BJH.

O volume de mesoporos calcula-se a partir da curva de dessorção da isoterma, enquanto que o volume total calcula-se no tramo de adsorção. Devido à ausência de platô quando p/po tende a 1,0 volume de mesoporos na argila natural dá um valor maior que o volume total (ver Tabela 3). Entretanto, isso não significa que o volume de mesoporos seja realmente maior que o volume total.

Comparando as duas isotermas, observa-se que o tratamento da argila natural com a solução oligomérica de Al dá lugar a um aumento considerável na quantidade de $\mathrm{N}_{2}$ adsorvido. Para p/po $=0,2$, a quantidade de $\mathrm{N}_{2}$ adsorvido pela argila natural é de 11,4 , enquanto que para a argila pilarizada é $65,5 \mathrm{~cm}^{3} / \mathrm{g}$, quer dizer, um aumento de $\sim 6$ vezes.

Na Tabela 3 apresentam-se os resultados de superfície específica e volume de poro calculados para as diferentes argilas pilarizadas preparadas.

Comparando a amostra PM15:15d com a Mnat, observa-se que os valores de áreas superficiais externas, que são a contribuição da superfície externa mais a de meso e macroporos, sofrem pouca variação. Quanto ao volume de poros é 12 vezes maior na amostra pilarizada, devido ao aumento do espaçamento basal. Em conseqüência deste aumento no volume de microporos, a área de microporos é $~ 12$ vezes maior. Esta área é a grande responsável

Tabela 2. Áreas BET dos materiais calculadas a partir da isoterma de adsorção de $\mathrm{N}_{2}$ obtida pelo CG2000 (calcinadas a $450{ }^{\circ} \mathrm{C}$ )

\begin{tabular}{cccc}
\hline Tempo de oligomerização & Concentração da suspensão de argila & Nomenclatura do material & $\mathrm{A}_{\mathrm{BET}}\left(\mathrm{m}^{2} / \mathrm{g}\right)$ \\
\hline $1 \mathrm{~h}$ & $1 \mathrm{~g} / 100 \mathrm{~mL}$ & PM100:0d & 156 \\
286 & PM100:6d & 209 \\
6 dias & $1 \mathrm{~g} / 100 \mathrm{~mL}$ & PM15:6d & 225 \\
\hline & $1 \mathrm{~g} / 15 \mathrm{~mL}$ & PM0:6d & 277 \\
& $1 \mathrm{~g} / 0 \mathrm{~mL}$ & PM100:15d & 306 \\
\hline
\end{tabular}


Tabela 3. Superfície específica e volume de poro calculados a partir da isoterma de adsorção para as amostras preparadas

\begin{tabular}{lcccccc}
\hline Amostra & $\mathrm{S}_{\text {BET }}\left(\mathrm{m}^{2} / \mathrm{g}\right)$ & $\mathrm{S}_{\text {micro }}\left(\mathrm{m}^{2} / \mathrm{g}\right)$ & $\mathrm{S}_{\text {Ext }}\left(\mathrm{m}^{2} / \mathrm{g}\right)$ & $\mathrm{V}_{\text {Total }}\left(\mathrm{cm}^{3} / \mathrm{g}\right)$ & $\mathrm{V}_{\text {micro }}\left(\mathrm{cm}^{3} / \mathrm{g}\right)$ & $\mathrm{V}_{\text {B̈H }}\left(\mathrm{cm}^{3} / \mathrm{g}\right)$ \\
\hline Mnat & 41 & 19 & 22 & 0,078 & 0,009 & 0,089 \\
PM15:15d & 255 & 236 & 19 & 0,147 & 0,110 & 0,046 \\
PM100:0d & 142 & 131 & 11 & 0,091 & 0,061 & 0,039 \\
\hline
\end{tabular}

pelo acréscimo de área total nos materiais pilarizados. Deve-se ter em conta que esta área não pode ser dita como real, ela é uma representação da quantidade de $\mathrm{N}_{2}$ adsorvido nos microporos.

A PM100:0d, quando comparada com a Mnat, possui a metade da área superficial externa e um volume de microporos 6,6 vezes maior; quando comparada com a PM15:15d, possui praticamente a metade do volume e de área de microporos, mesmo o $\mathrm{d}_{001}$ sendo semelhante. Isto confirma os resultados obtidos anteriormente na análise de adsorção de $\mathrm{N}_{2}$ pelo CG2000.

A Tabela 4 compara as áreas BET obtidas das isotermas de adsorção de $\mathrm{N}_{2}$ empregando CG2000 e ASAP2400.

Observam-se diferenças nas áreas obtidas para a mesma amostra, que podem ser devidas aos diferentes pré-tratamentos utilizados. Entretanto, a área da PM100:0d permaneceu aproximadamente a metade do valor de PM15:15d

Tabela 4. Superfícies específicas calculadas pelas isotermas de adsorção empregando o CG2000 e o ASAP2400

\begin{tabular}{lcc}
\hline Amostra & ASAP2400 $\left(\mathrm{m}^{2} / \mathrm{g}\right)$ & CG2000 $\left(\mathrm{m}^{2} / \mathrm{g}\right)$ \\
\hline Mnat & $41^{*}$ & $33^{+}$ \\
PM15:15d & $255^{*}$ & $306^{+}$ \\
PM100:0d & $142^{\#}$ & $156^{+}$ \\
\hline
\end{tabular}

* pré-tratadas a $200{ }^{\circ} \mathrm{C}$ por $4 \mathrm{~h}$; + pré-tratadas a $250{ }^{\circ} \mathrm{C}$ por $2 \mathrm{~h}$; \# pré-tratadas a $300{ }^{\circ} \mathrm{C}$ por $3 \mathrm{~h}$

\section{Testes catalíticos}

Foram realizados testes de atividade catalítica com o cumeno para avaliar as propriedades de craqueamento dos materiais.

Testes catalíticos conduzidos com areia e com o reator vazio não proporcionaram a reação de craqueamento do cumeno.

A argila natural calcinada praticamente não teve atividade catalítica para a reação do craqueamento do cumeno. Foram observados traços de benzeno nos primeiros $10 \mathrm{~min}$ do teste.

A Figura 7 ilustra o teste do craqueamento do cumeno para a amostra PM15:6d. Observa-se que no início do teste tem-se aproximadamente $33 \%$ de benzeno, e depois de $1 \mathrm{~h}$ a porcentagem de benzeno reduz-se à metade, evidenciando uma rápida perda de atividade. Para tempos maiores que $1 \mathrm{~h}$, a perda de atividade torna-se cada vez menor e a porcentagem de benzeno começa a permanecer constante. Após $3 \mathrm{~h}$ do início do teste, tem-se $13 \%$ de benzeno produzido.

Para todos os testes o material após a reação torna-se preto, devido supostamente à formação de coque e desativação do material.

O processo de pilarização efetivamente produziu modificações na argila natural, que resultaram em atividade de craqueamento.

Segundo Tung e Mcninch ${ }^{12}$, o tipo de produtos obtidos no craqueamento de cumeno, benzeno e propileno é característico de reação que ocorre por um mecanismo iônico utilizando sítios de Brönsted. Não foram detectados produtos característicos de sítios de Lewis. Ming-Yuan et al. ${ }^{13}$ asseguraram que os pilares são a principal fonte de acidez do tipo de Lewis e que os sítios de Brönsted

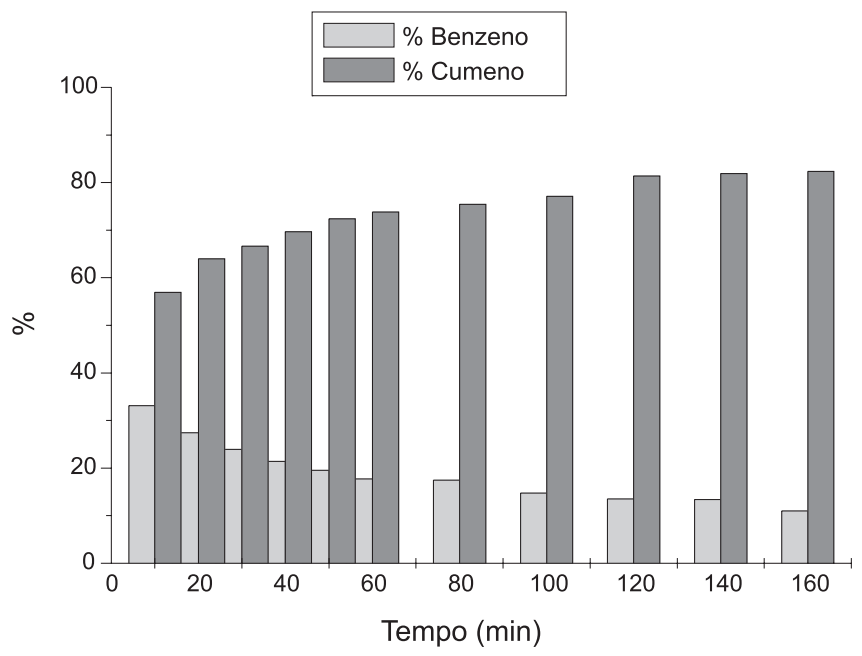

Figura 7. Atividade catalítica para o craqueamento do cumeno sobre a PM15:6d

estariam associados apenas aos grupos - $\mathrm{OH}$ da estrutura da argila. Para temperaturas de calcinação acima de $400{ }^{\circ} \mathrm{C}$, Poncelet e Schultz ${ }^{14}$ mostraram que os sítios de Brönsted da argila são os responsáveis pelo craqueamento de cumeno. Estes resultados suportam que a atividade determinada neste trabalho é decorrente da argila e não dos pilares de alumínio introduzidos durante o processo de pilarização.

Para comparação dos desempenhos das argilas pilarizadas foram utilizados dois parâmetros: (i) rendimento médio inicial correspondente à quantidade de benzeno produzida nos primeiros 60 min do teste e (ii) rendimento residual após 140 min correspondente a quantidade de benzeno produzida num intervalo de $20 \mathrm{~min}$.

As Figuras 8 e 9 mostram estes parâmetros. De forma geral, não se observam grandes variações de resultados. A amostra preparada com tempo de oligomerização de $1 \mathrm{~h}$, MP100:0d, que resultou nas maiores quantidades de Al incorporado, menor espaçamento basal e menor área superficial BET (aproximadamente $150 \mathrm{~m}^{2} / \mathrm{g}$ ), proporcionou quantidades de benzeno equivalentes a amostras com áreas de até $300 \mathrm{~m}^{2} / \mathrm{g}$. A hipótese levantada anteriormente para explicar estas diferenças de áreas BET foi que a MP100:0d adsorve apenas uma camada de $\mathrm{N}_{2}$, enquanto que nos outros materiais com áreas superiores haveria diferentes graus de adsorção em dupla camada. O diâmetro molecular do cumeno é $6,7 \AA^{15}$, devido a este tamanho seria possível a formação de uma monocamada em todos os materiais pilarizados. $\mathrm{O}$ espaçamento intermolecular é maior nos materiais que obtiveram áreas maiores, mas a superfície da argila, responsável pelos sítios de Brönsted, é a mesma e, conseqüentemente, as conversões de cumeno seriam semelhantes.

O aumento da diluição da suspensão de argila, que proporciona uma maior incorporação de alumínio e um aumento no espaçamento basal, tende a reduzir a quantidade de benzeno produzida. O aumento do alumínio incorporado estaria propiciando uma maior acidez de Lewis e não de Brönsted. A acidez de Brönsted pode diminuir como o alumínio incorporado, uma vez que este ao 


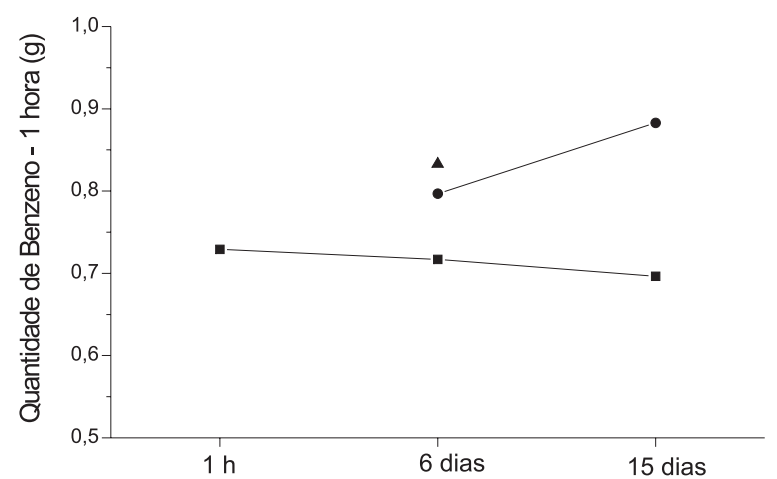

Figura 8. Rendimento médio inicial (correspondente à quantidade de benzeno produzida nos primeiros 60 min do teste) em função da concentração da suspensão de argila e tempo de envelhecimento da solução pilarizante $(\mathbf{\Delta}=$ $1 \mathrm{~g} / 0 \mathrm{~mL} ; \mathbf{O}=1 \mathrm{~g} / 15 \mathrm{~mL} ; \mathbf{\square}=1 \mathrm{~g} / 100 \mathrm{~mL}$ )

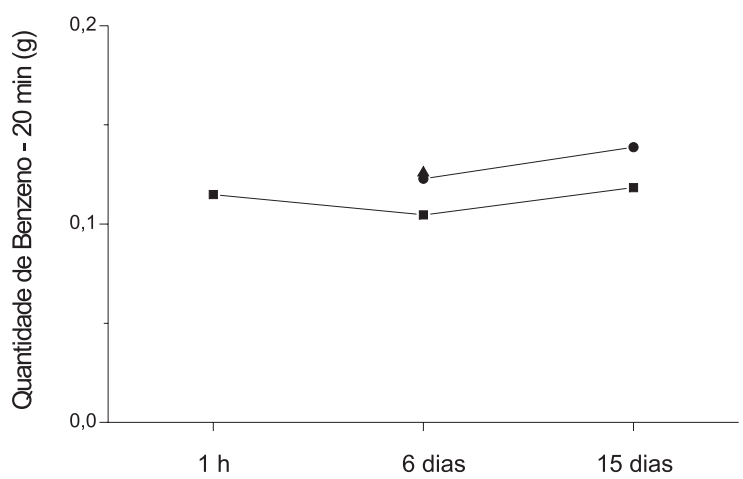

Figura 9. Rendimento residual após $140 \mathrm{~min}$ (correspondente à quantidade de benzeno produzida num intervalo de 20 min) em função da concentração da suspensão de argila e tempo de envelhecimento da solução pilarizante $(\mathbf{\Lambda}=1 \mathrm{~g} / 0 \mathrm{~mL} ; \boldsymbol{O}=1 \mathrm{~g} / 15 \mathrm{~mL} ; \mathbf{\square}=1 \mathrm{~g} / 100 \mathrm{~mL})$

ocupar parte da superfície da argila não deixaria esta disponível para a reação. Um maior espaçamento basal, neste caso, não modifica o acesso das moléculas de cumeno. Paralelamente, a interação das moléculas de cumeno com a superfície pode aumentar com a diminuição do espaçamento basal, o que conduziria a conversões de craqueamento maiores. Ambos efeitos parecem suportar as tendências de conversão observadas.

Estes resultados indicam que as condições ótimas para maximizar as incorporações de oligômeros e o espaçamento basal não são necessáriamente as melhores para a aplicação desejada. Deve- se ressaltar, no entanto, que no presente caso, a conversão de cumeno foi utilizada para caracterizar propriedades catalíticas e tamanho de poros do material pilarizado e não como a aplicação final desejada. O craqueamento de outras moléculas pode conduzir a resultados diferentes.

\section{CONCLUSÃO}

O processo de pilarização proporcionou materiais com maiores conteúdos de $\mathrm{Al}$ e um aumento do espaçamento basal de 9,7 a 18,5 Å, indicando a formação de um material com suas lâminas separadas por pilares. Esta separação das lâminas proporciona uma microporosidade adicional e, conseqüentemente, um aumento da superfície específica de 41 a até $\sim 300 \mathrm{~m}^{2} / \mathrm{g}$.

O aumento da diluição da suspensão de argila promove a formação de produtos com maiores espaçamentos basais e quantidades de alumínio incorporado. Soluções pilarizantes envelhecidas por $1 \mathrm{~h}$ conduziram a produtos com espaçamento basal cerca de $0,6 \AA$ menores que aquelas envelhecidas por 6 e 15 dias e áreas superficiais BET 50\% menores. As conversões no craqueamento do cumeno são similiares em todas as argilas pilarizadas independentemente das variações de áreas superficiais. Pequenas reduções na atividade de craqueamento correlacionam-se com aumentos do espaçamento basal e da quantidade de alumínio incorporado.

\section{REFERÊNCIAS}

1. Santos, P. S.; Coelho, A. C. V.; B. Téc. Petrobrás 1988, 31, 143.

2. Burch, R.; Catal. Today 1988, 2, 185.

3. Mitchell, I. V.; Pillared Layered Structures - Current Trends and Applications, Elsevier Applied Science: London, 1990.

4. Pergher, S. B. C.; Tese de Mestrado, Universidade Estadual de Maringá, Maringá, Brasil, 1993.

5. Pergher, S. B. C.; Tese de Doutorado, Universidade Politécnica de Valencia - Instituto de Tecnologia Química, Valencia, Espanha, 1997.

6. Pergher, S. B. C.; Corma, A.; Fornés, V.; Quim. Nova 1999, 22, 693.

7. Luna, F. J.; Schuchardt, U.; Quim. Nova 1999, 22, 104.

8. Leite, S. Q. M.; Dieguez, L. C.; San Gil, R. A. S.; Menezes, S. M. C. de; Quim. Nova 2000, 23, 149.

9. Pergher, S. B. C.; Corma, A. ; Fornes, V.; Quim. Nova 1999, 22, 649.

10. Breck, D. W.; Zeolites Molecular Sieves, John Wiley \& Sons: New York, 1974.

11. Gregg, S. I.; Sing, K. S. W.; Adsorption, Surface Area and Porosity, Academic Press: London, 1982.

12. Tung, S. E.; Mcininch, E.; J. Catal. 1965, 4, 586.

13. Ming-Yuan, H.; Zhoghui, L.; Enze, M.; Catal. Today 1988, 2, 321.

14. Pocelet, G.; Schultz, A.; Em Chemical Reaction in Organic and Inorganic Constrained Systems; Selton, R., ed.; 1986, p. 165-178.

15. Satterfield, C. N.; Heterogeneus Catalysis in Practice, Mc. Graw Hill, 1980. 